\title{
The Surveillance Window - Contextualizing Data Streams
}

\author{
Kirsten McCabe*, Lauren Castro, Mac Brown, William Daniel, Eric Nick Generous, Kristen \\ Margevicius and Alina Deshpande
}

Los Alamos National Laboratory, Los Alamos, NM, USA

\section{Objective}

The goal of this project is the evaluation of data stream utility in integrated, global disease surveillance. This effort is part of a larger project with the goal of developing tools to provide decision-makers with timely information to predict, prepare for, and mitigate the spread of disease.

\section{Introduction}

Los Alamos National Laboratory has been funded by the Defense Threat Reduction Agency to determine the relevance of data streams for an integrated global biosurveillance system. We used a novel method of evaluating the effectiveness of data streams called the "surveillance window". The concept of the surveillance window is defined as the brief period of time when information gathered can be used to assist decision makers in effectively responding to an impending outbreak. We used a stepwise approach to defining disease specific surveillance windows;

1. Timeline generation through historical perspectives and epidemiological simulations.

2. Identifying the surveillance windows between changes in "epidemiological state" of an outbreak.

3. Data streams that are used or could have been used due to their availability during the generated timeline are identified. If these data streams fall within a surveillance window, and provide both actionable and non-actionable information, they are deemed to have utility.

\section{Methods}

Figure 1 shows the overall approach to using this method for evaluating data stream types. Our first step was identifying a list of priority diseases to build surveillance windows for and our primary sources were our SME panel, CDC priorities, as well as DOD priorities. We also conducted a literature review to support our selection of diseases. We ensured that there was representation of human, animal and plant diseases and there was enough data available for selected outbreaks to facilitate evaluation of all data stream types identified. We then selected representative outbreaks for diseases to generate a timeline for defining surveillance windows. Surveillance windows were then defined (based on four specific biosurveillance goals developed by LANL) and information for applicable data streams was collected for the duration of the outbreak. A data stream was deemed useful if it was determined to be available within the defined surveillance window. In addition, evaluation of the ideal use case of the data streams was performed. In essence, if used more effectively could this data stream provide greater support to understanding, detection, warning or management of disease outbreaks or event situations?

\section{Results}

Results presented in this abstract are from retrospective analyses of historical outbreaks selected as being representative of FMD,
Ebola, Influenza and E.coli. Graphs indicating case counts and geographical spread were combined and a timeline was created to determine the length of time between changes in "epidemiological state" that defined various surveillance windows. This timeline was then populated with durations when data streams were used during the outbreak. Results showed varying surveillance windows times are dependent on disease characteristics. In turn, epidemiology of the disease affected the occurrence of data streams on the timeline.

\section{Conclusions}

Surveillance window based evaluation of data streams during disease outbreaks helped identify data streams that are of significance for developing an effective biosurveillance system. Some data streams were identified to have high utility for early detection and early warning regardless of disease, while others were more disease and operations specific. This work also identified data streams currently not in use that could be exploited for faster outbreak detection. Key useful data streams that are underlying to all disease categories and thus important for integration into global biosurveillance programs will be presented here.

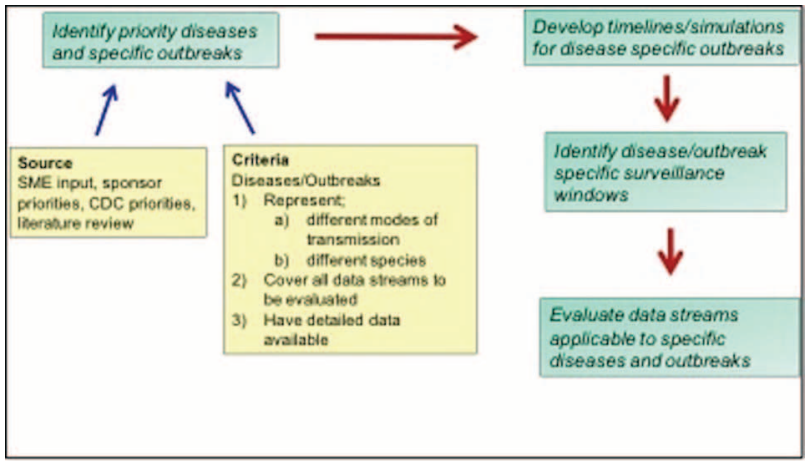

Figure 1: Overall approach to surveillance window based evaluation

\section{Keywords}

Surveilliance Windows; Data streams; Biosurveilliance

\section{Acknowledgments}

This project is supported by the Chemical and Biological Technologies Directorate Joint Science and Technology Office (JSTO), Defense Threat Reduction Agency (DTRA).

*Kirsten McCabe

E-mail: kjmccab@lanl.gov 